\title{
$\beta$-Cyclodextrin functionalized molybdenum disulfide quantum dots as nanoprobe for sensitive fluorescent detection of parathion-methyl
}

\author{
Yinhui $\mathrm{Yi}^{\mathrm{a}}{ }^{\mathrm{a}, \mathrm{d}, * *}$, Wei Zeng ${ }^{\mathrm{a}}$, Gangbing Zhu ${ }^{\text {a,c, }, * *}$ \\ ${ }^{a}$ School of the Environment and Safety Engineering, Jiangsu University, Zhenjiang, 212013, PR China \\ ${ }^{\mathrm{b}}$ State Key Laboratory of Chemo/Biosensing and Chemometrics, Hunan University, Changsha, 410082, PR China \\ ${ }^{\mathrm{c}}$ Key Laboratory of Coastal Environmental Processes and Ecological Remediation, Yantai Institute of Coastal Zone Research, Chinese Academy of Sciences, PR China \\ ${ }^{\mathrm{d}}$ Key Laboratory for Analytical Science of Food Safety and Biology, Ministry of Education, PR China
}

\section{A R T I C L E I N F O}

\section{Keywords:}

Parathion-methyl

Quantum dots

Cyclodextrin

Fluorescent sensing

Organophosphorus pesticides

Probe

\begin{abstract}
A B S T R A C T
Through modifying molybdenum disulfide quantum dots ( $\mathrm{MoS}_{2}$ QDs) with 3-aminophenyl boronic acid and functionalizing further with hydropropyl- $\beta$-cyclodextrin $(\beta-C D)$, a novel nanoprobe based on $\beta$-CD functionalized $\mathrm{MoS}_{2}$ QDs ( $\beta$-CD-MoS 2 QDs) was developed for the fluorescent detection of parathion-methyl (MP). $\beta-\mathrm{CD}-\mathrm{MoS}_{2}$ QDs was characterized with various technologies including transmission electron microscopy, X-ray photoelectron spectroscopy, fluorescence and UV-Vis absorption spectra. As for MP detection, MP was hydrolyzed to pnitrophenol (p-NP) under the alkaline conditions, and p-NP can enter into the $\beta$-CD cavity due to the host-guest recognition capability of $\beta-C D$, which then results the fluorescence quenching of nanoprobe. Based on this principle, an enzyme-free fluorescence sensing platform were constructed for MP. Under the optimal conditions, $\beta-\mathrm{CD}-\mathrm{MoS}_{2}$ QDs nanoprobe exhibits wide detection scope (0.01-18.0 ppm) and low detection limits (3.3 ppb) for MP detection. In addition, the nanoprobe has excellent selectivity for MP, and it can be applied to detect MP in real samples.
\end{abstract}

\section{Introduction}

Organophosphorus pesticides (OPs) are a cluster of organic pesticides which have been extensively utilized in agriculture due to the excellent ability to control pests and plant diseases [1-3]. The residues substances of OPs have been found to cause organ dysfunction, cancer disease and fetal anomaly even at very low concentration. Parathion-methyl (MP) is a typical OPs which incline to accumulate in food and water because of its bioaccumulation and low solubility [4]. According to the guidelines for drinking water quality ruled by World Health Organization, the concentration of MP in agricultural water ranges up to $1.7 \mathrm{nM}$ increasing during summer months. Environmental Protection Agency (EPA) also regulates that the safe level of MP in drinking water is as follows: $0.11 \mu \mathrm{M}$ for longer term exposure of children, $1.1 \mu \mathrm{M}$ for $1 \mathrm{~d}$ or $10 \mathrm{~d}$ of exposure for children; and $7.6 \mathrm{nM}$ for the adults lifetime exposure [5,6]. Therefore, developing sensitive detection method for MP is of great significance to ensure environment and food safety.

During the past years, several analytical techniques have been designed to detect MP pesticide, such as gas chromatography, gas chromatography-mass spectrometry, chemiluminescence, surfaceenhanced Raman spectroscopy [7], electrochemical analysis [8] and enzyme-linked immunosorbent assay [9]. Although the aforementioned methods are very important for detecting MP, their real applications are still restricted by some unavoidable shortcomings such as complex pre-treatment processes, high-cost and requiring skillful operation [10, 11]. Recently, some interesting works have been reported for detecting MP based on the fluorescence nanoprobe due to its unique advantages on cost, convenience and sensitivity. For instance, Xu et al. [12] established a fluorescence sensor based on the catalytic reaction of organophosphorus hydrolase for MP detection. Huang et al. [13] construct a biosensor based on acetylcholinesterase enzymatic reaction for OPs assay. However, most of the developed approaches for MP detection based on fluorescence nanoprobe generally depend on the catalytic or inhibitory effect of the enzyme, and the enzyme is relatively expensive and easily inactivated with short service life. For resolving this problem in MP detection, there are a few sensor arrays based on enzyme-free fluorescent nanoprobe have been proposed most recently [14], but

\footnotetext{
* Corresponding author. School of the Environment and Safety Engineering, Jiangsu University, Zhenjiang, 212013, PR China.

** Corresponding author. School of the Environment and Safety Engineering, Jiangsu University, Zhenjiang, 212013, PR China.

E-mail addresses: yyh1108@ujs.edu.cn (Y. Yi), zhgb1030@ujs.edu.cn (G. Zhu).
} 
developing enzyme-free nanoprobe for the fluorescent detection of MP with high selectivity and sensitivity is still a challenge.

Transition metal chalcogenides attracted recently widespread attentions as a graphene-analogous two-dimensional nanomaterial which can exhibit uncommon and unique electronic, mechanical, optical, chemical and thermal properties $[15,16]$. Among these transition metal chalcogenides nanomaterials, molybdenum disulfide $\left(\mathrm{MoS}_{2}\right)$ has been exploited largely in the past years with important potential application as novel optical sensors, optoelectronic devices, batteries, transistors and catalysts. As is well-known, the optical properties from semiconductors relay mainly on the electronic band structure of nanomaterial [17]. When the indirect band gap from bulk $\mathrm{MoS}_{2}$ (about 1.2 eV) transfer to direct band gap of monolayer $\mathrm{MoS}_{2}$ (about $1.8 \mathrm{eV}$ ), the photoluminescent behavior from $\mathrm{MoS}_{2}$ can be aroused [18,19]. In addition, when the lateral size $\mathrm{MoS}_{2}$ nanosheets decrease to less than 10 $\mathrm{nm}$ to form $\mathrm{MoS}_{2}$ quantum dots $\left(\mathrm{MoS}_{2}\right.$ QDs), the formed $\mathrm{MoS}_{2}$ QDs can show strong fluorescence owing to the quantum confinement and edge effects, and $\mathrm{MoS}_{2}$ QDs exhibit many unique superiorities such as low toxicity, environmentally friendly and easily functionalized [20]. Thus $\mathrm{MoS}_{2}$ QDs have been widely applied in fluorescence determination of metal ion [21], hyaluronidase, pyrophosphate [20,22], etc. [23]. However, most fluorescence sensing platforms based on pure $\mathrm{MoS}_{2}$ QDs usually exist several defects such as low selectivity and poor anti-interference [24], designing or bring in a new multifunctional molecule which has benign effects on the selectivity and sensitivity as well as other sensing performances of $\mathrm{MoS}_{2} \mathrm{QD}$ is thus highly interesting and important technologically.

$\beta$-Cyclodextrin ( $\beta$-CD), a cyclic oligosaccharide consisted of 7 glucose units, is a well-known molecular host which is capable of including various organic molecules inside its cavity to form host-guest inclusion complex [25]. Because of its hydrophobic inner cavity, hydrophilic surface structure and molecular recognition property, $\beta$-CD has been widely used to develop various sensing platforms via host-guest interaction [26]. Thus, it's believed that the combination of the strong fluorescence properties from $\mathrm{MoS}_{2}$ QD and high molecular recognition capabilities from $\beta-C D$ can offer a novel pathway to construct highly sensitive and selective sensing platforms for different analytes including MP.

The previous studies demonstrated that MP can be hydrolyzed to pnitrophenol (p-NP) under alkaline conditions [27], and p-NP can enter into the $\beta$-CD cavity via host-guest recognize between $\beta$-CD and $\mathrm{p}-\mathrm{NP}$ [28]. Inspired by this insight, it's confirmed that the alliance of $\mathrm{MoS}_{2}$ QDs and $\beta$-CDs can develop selective and sensitive fluorescent nanoprobe for MP via measuring the signaling changes produced by the entrance of $\mathrm{p}-\mathrm{NP}$ into $\beta$-CD cavity. In the present study, $\beta$-CD functionalized $\mathrm{MoS}_{2}$ QDs ( $\beta$-CD-MoS 2 QDs) was synthesized firstly and then used as a novel enzyme-free fluorescent nanoprobe for the detection of MP. As shown in Scheme S1, 3-aminophenylboronic acid (APBA) modified $\mathrm{MoS}_{2}$ QDs was formed firstly via amidation reaction, which then couple with hydroxypropyl- $\beta-\mathrm{CD}$ to produce $\beta-\mathrm{CD}-\mathrm{MoS}_{2}$ QDs. Under the alkaline conditions, the target MP can be hydrolyzed to p-NP and sequentially the formed $\mathrm{p}$-NP enters into $\beta$-CD cavity via host-guest recognize between $\beta$-CD and $\mathrm{p}-\mathrm{NP}$, which results the fluorescence from $\beta$-CD-MoS 2 QDs are effectively quenched (Scheme 1). Based on this principle, an effective and reliable sensing platform was constructed for MP. The experimental results demonstrated that the detection method based on $\beta$-CD-MoS ${ }_{2}$ QDs probe for MP has high selectivity and sensitivity as well as wide linear range and low detection limit. it's believed the developed proposal has important potential applications for MP analysis and the produced $\beta$-CD-MoS 2 QDs nanoprobe has wide applications in the optical sensing.

\section{Experiment}

\subsection{Materials and regents}

Sodium molybdate dihydrate $\left(\mathrm{Na}_{2} \mathrm{MoO}_{4} \cdot 2 \mathrm{H}_{2} \mathrm{O}\right)$, 1-ethyl-3-(3-dimethylaminopropyl)carbodiimide (EDC. $\mathrm{HCl}$ ), APBA, glutathione, p-NP, hydropropyl- $\beta-\mathrm{CD}, \mathrm{N}$-hydroxysuccinimide (NHS), MP and other pesticides were purchased from Aladdin Industrial Co. Ltd. (Shanghai, China).

\subsection{Characterization}

The fluorescence spectrums were measured with F-7000 Spectrofluorometer (Shimadzu, Japan). UV-Vis absorption spectra were carried out on UV-Vis 2600 spectrophotometer (Shimadzu, Japan). X-ray photoelectron spectroscopy was measured on a K-Alpha 1063 (Thermo Fisher, U. K.). Transmission electron microscopy (TEM) was obtained using a JEM-3010 (Joel, Japan). The fluorescence lifetime was acquired by Horiba-Jobin-Yvon Fluorolog-3 spectrofluorometer (Horiba, Japan). Purification of $\beta$-CD-MoS 2 QDs was performed using a centrifuge $5804 \mathrm{R}$ (Eppendorf, Germany).

\subsection{Preparing $\mathrm{MoS}_{2}$ QDs}

$\mathrm{MoS}_{2}$ QDs were synthesized via a modified process reported in previous work [15]. $\mathrm{Na}_{2} \mathrm{MoO}_{4} \cdot 2 \mathrm{H}_{2} \mathrm{O}(0.125 \mathrm{~g})$ was dissolved in ultrapure water $(12.5 \mathrm{~mL})$ and adjusting its $\mathrm{pH}$ value to 6.5 with $0.1 \mathrm{M} \mathrm{HCl}$, then, the glutathione $(0.215 \mathrm{~g})$ and ultrapure water $(25 \mathrm{~mL})$ were added. After

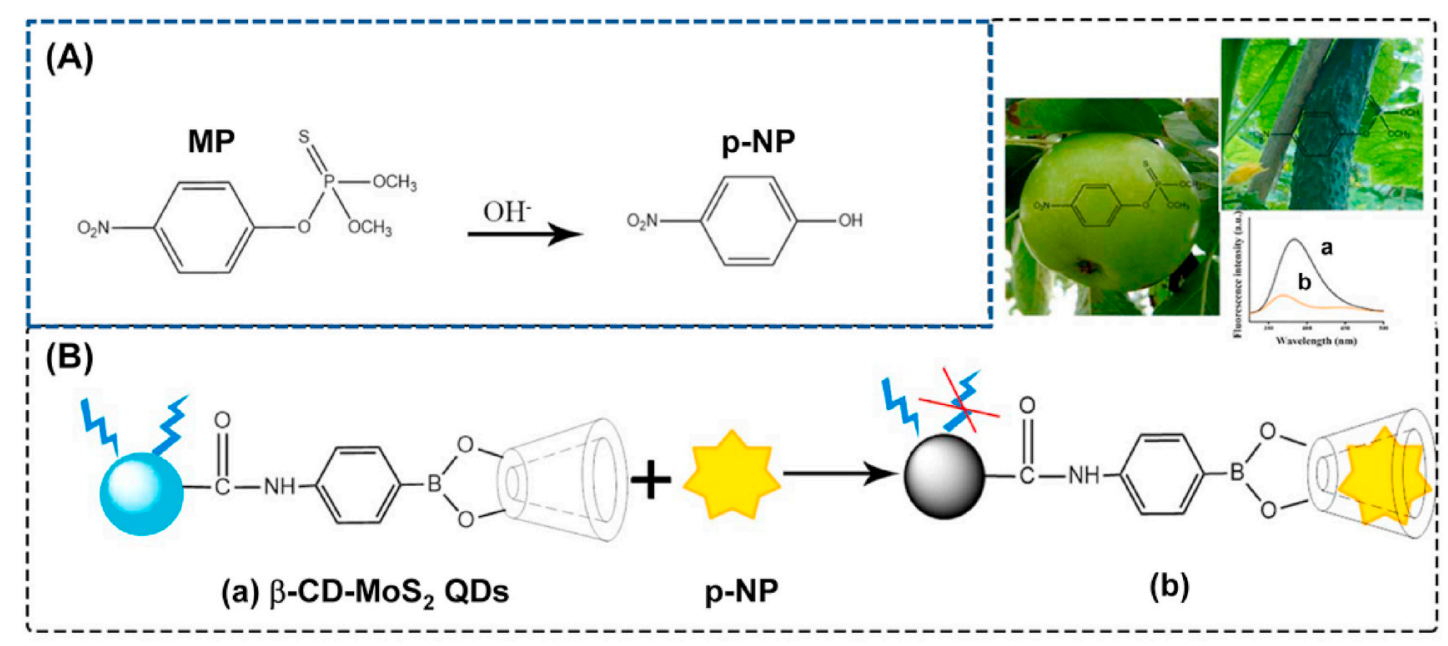

Scheme 1. Schematic illustrations for (A) MP hydrolysis and (B) fluorescent sensing MP based on $\beta$-CD-MoS ${ }_{2}$ QDs nanoprobe through host-guest recognition. 
sonicating for about $10 \mathrm{~min}$, the formed mixture solution was transferred into an autoclave $(50.0 \mathrm{~mL})$ and hydrothermally treated for one day at $200{ }^{\circ} \mathrm{C}$. After cooled to room temperature, the large particles consisted of reaction solutions were removed through centrifugation. The pure $\mathrm{MoS}_{2}$ QDs solution was stored in $4{ }^{\circ} \mathrm{C}$.

\subsection{Synthesis of $\beta-C D-M o S_{2} Q D s$}

For synthesizing $\beta$-CD-MoS 2 QDs, the produced above $\mathrm{MoS}_{2}$ QDs $(20.0 \mathrm{~mL}$ ) were added into $10.0 \mathrm{~mL}$ of ultrapure water, adjusting the $\mathrm{pH}$ value of the solution to 4.0. EDC. $\mathrm{HCl}(0.2 \mathrm{~g})$ and NHS $(0.2 \mathrm{~g})$ were put into the $\mathrm{MoS}_{2}$ QDs solution and stirred continuously for $30 \mathrm{~min}$. Next, APBA $(0.25 \mathrm{~g})$ was added into the solution and heated at $100{ }^{\circ} \mathrm{C}$ for $12 \mathrm{~h}$. Adjusting the $\mathrm{pH}$ value of the resulting solution to 9.0 with $\mathrm{NaOH}$ solution, and adding $0.35 \mathrm{~g}$ hydroxypropyl- $\beta$-CD into the solution for functionalizing $\mathrm{MoS}_{2}$ QDs, the resulted mixed solution was heated continuously for $5 \mathrm{~h}$ at $100{ }^{\circ} \mathrm{C}$. Finally, after purifying the obtained solution with 1000 Da dialysis bag for removing excessive EDC, NHS, APBA and $\beta$-CD, $\beta$-CD-MoS 2 QDs was obtained successfully.

\subsection{Fluorescence detection of $M P$ by $\beta-C D-M o S_{2} Q D s$}

The analysis of MP was performed through the following steps. At first, MP $(100.0 \mu \mathrm{L})$ with a definite concentration was added into the solution of $\mathrm{NaOH}(35.0 \mathrm{mM})$ and incubated at $60{ }^{\circ} \mathrm{C}$ for $20 \mathrm{~min}$ to produce $\mathrm{p}-\mathrm{NP}$ via hydrolytic action. Then, $10.0 \mu \mathrm{L}$ of $\beta$-CD-MoS ${ }_{2} \mathrm{QDs}$ was added into this mixed solution after adjusting its $\mathrm{pH}$ value to neutral with $5.0 \mathrm{~mL}$ PBS ( $\mathrm{pH} \mathrm{6.0)}$ ). For achieving the fluorescence of MP, the fluorescence intensities at the excitation wavelength of $295 \mathrm{~nm}$ was measured.

\subsection{Real samples detection}

After crushing the edible portion of the real samples, a total of $25.0 \mathrm{~g}$ of each sample was mixed with acetonitrile solution $(50.0 \mathrm{~mL})$ and then the mixture was filtered. The collected solution was dried on a water bath and subsequently dissolved in a methanol solution. Next, MP with various concentrations were added to the treated samples and incubating at $60{ }^{\circ} \mathrm{C}$ for $20 \mathrm{~min}$ with $\mathrm{NaOH}$ solution $(35.0 \mathrm{mM})$ to produce pNP via hydrolytic action. Then, the hydrolyzed samples were added to $5.0 \mathrm{~mL}$ PBS and mixed with $\beta$-CD-MoS 2 QDs $(10 \mu \mathrm{L})$, the fluorescence intensity was measured at the excitation wavelength of $295 \mathrm{~nm}$.

\section{Results and discussion}

\subsection{Characterization of $\beta-C D-M o S_{2} Q D s$}

The morphology and distribution of the obtained $\beta$-CD-MoS2 QDs

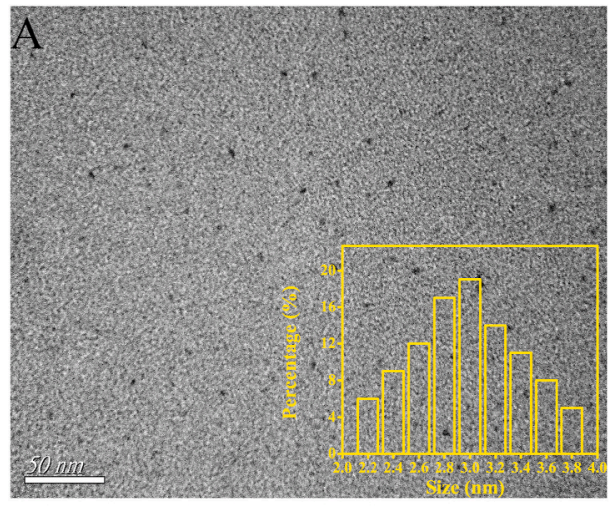

were characterized by TEM, as shown in Fig. 1A, the picture displayed the uniformly dispersed of $\beta$-CD-MoS ${ }_{2}$ QDs with an average diameter of $\sim 3 \mathrm{~nm}$, which is in consistent with that of original $\mathrm{MoS}_{2}$ QDs (Fig. S1). XPS spectrum was determined the surface element composition of $\beta$-CD$\mathrm{MoS}_{2}$ QDs, and the result are shown in Fig. 1B. The binding energy signal at $395.2 \mathrm{eV}$ corresponded to $\mathrm{N}-\mathrm{C}=\mathrm{O}$, which can confirmed the formation of an amide bond [29]. The peaks at $191.9 \mathrm{eV}$ and $226.8 \mathrm{eV}$ represented to B (-B-O-), and Mo, respectively [30,31]. Fig. S2A shows that the Mo $3 \mathrm{~d}$ spectrum is deconvoluted into three peaks, which are located

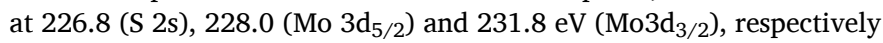
$[32,33]$. From Fig. S2B, it can be found that the $S 2 p$ spectrum displayed $\mathrm{S} 2 \mathrm{p}_{3 / 2}$ and $\mathrm{S} 2 \mathrm{p}_{1 / 2}$ at $163 \mathrm{eV}^{-1}$ and $164 \mathrm{eV}^{-1}$, respectively, and the other two peaks in the spectrum are belong to $\mathrm{S}_{2} \mathrm{O}_{3}^{2-}$ group [19]. In Fig. S2C, the $\mathrm{B} 1 \mathrm{~s}$ spectrum shows two peaks appear at $191.0 \mathrm{eV}^{-1}$ for $\mathrm{B}-\mathrm{C}, 191.9$ $\mathrm{eV}^{-1}$ for $\mathrm{B}-\mathrm{O}[34,35]$. The Fourier transform infrared spectroscopy (FT-IR) of $\beta$-CD-MoS ${ }_{2}$ QDs was exhibited in Fig. S3, It's noted that the $\beta$-CD-MoS 2 QDs shows strong peaks of $\mathrm{MoS}_{2}$ QDs at $3400 \mathrm{~cm}^{-1}, 1640$ $\mathrm{cm}^{-1}$ and $1036 \mathrm{~cm}^{-1}$ ascribed to $\mathrm{N}-\mathrm{H}$ stretching vibration, $\mathrm{N}-\mathrm{H}$ bending vibration and $\mathrm{B}-\mathrm{O}-\mathrm{H}$ vibration, respectively. Meanwhile, the typical absorption features of $\beta$-CD for the coupled $\mathrm{C}-\mathrm{O}-\mathrm{C}$ stretching/O-H bending vibrations at $1146 \mathrm{~cm}^{-1}$ and $\mathrm{CH}_{2}$ stretching vibrations at 2915 $\mathrm{cm}^{-1}$ can also be observed in $\beta$-CD-MoS 2 QDs. The results indicate that $\beta$-CD molecules were successfully linked covalently to $\mathrm{MoS}_{2}$ QDs to produce $\beta$-CD- $\mathrm{MoS}_{2}$ QDs nanoprobe through phenylboronic acid and amide bond.

The UV-vis displayed the different absorption spectrum between $\beta-\mathrm{CD}-\mathrm{MoS}_{2}$ QDs and $\mathrm{MoS}_{2}$ QDs. The $\beta$-CD-MoS 2 QDs shows two remarkable absorption peaks at $238 \mathrm{~nm}$ and $289 \mathrm{~nm}$, where no absorption peaks can be found for $\mathrm{MoS}_{2}$ QDs (Fig. S4) [30]. Moreover,

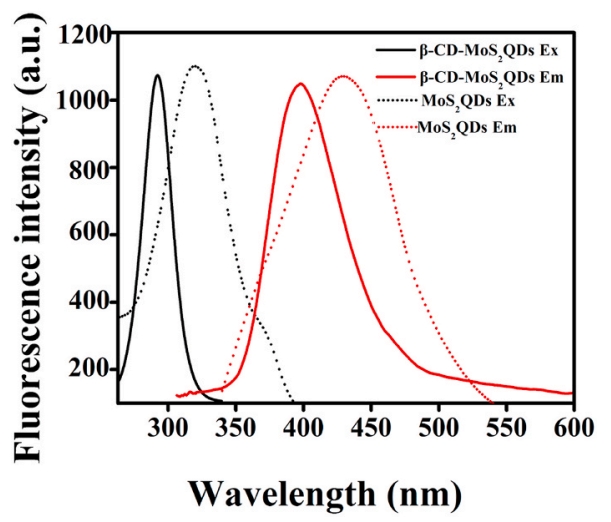

Fig. 2. Fluorescence excitation and emission spectra of $\beta-\mathrm{CD}-\mathrm{MoS}_{2}$ QDs (solid line) and $\mathrm{MoS}_{2}$ QDs (dashed line).

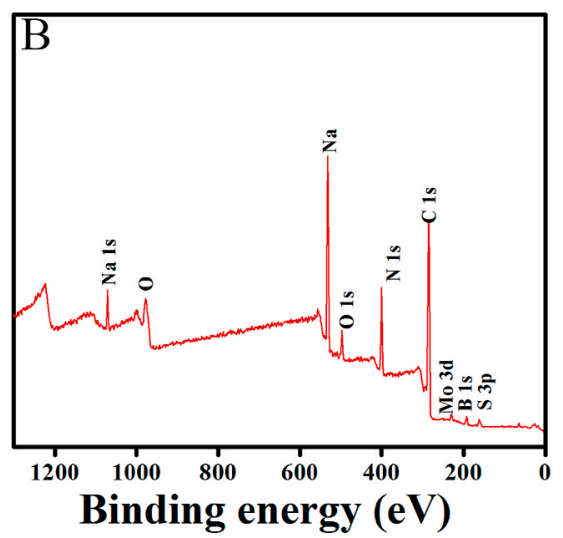

Fig. 1. (A) The TEM image and size distribution of $\beta-C D-M o S_{2}$ QDs; (B) the XPS spectrum of $\beta-C D-M o S_{2}$ QDs. 
Fig. 2 reveals that the excitation and emission peaks of $\beta$-CD-MoS ${ }_{2}$ QDs are greatly shifted compared to that of $\mathrm{MoS}_{2}$ QDs, suggesting that the $\beta$-CD covalently link to the surface of APBA functionalized $\mathrm{MoS}_{2}$ QDs through coupled effect between boronic acids and cis-diols. In summary, by comparing the XPS, UV-vis and fluorescence spectrum of $\beta$-CD-MoS QDs and $\mathrm{MoS}_{2}$ QDs, it's ensured that the $\beta$-CD-MoS 2 QDs fluorescent nanoprobe was successfully synthesized.

To further explore the stability of the as-prepared fluorescent nanoprobe, the fluorescence intensity of $\beta$-CD-MoS 2 QDs exposed to daylight for $8 \mathrm{~h}$ and in different concentrations of $\mathrm{NaCl}$ solution was measured. As illustrated in Fig. S5, no significant changes observed in the fluorescence intensity of $\beta$-CD-MoS $S_{2}$ QDs under irradiation of daylight for $8 \mathrm{~h}$ or in a highly concentrated solution of $\mathrm{NaCl}$. These results illustrate the synthesized $\beta$-CD-MoS 2 QDs possess excellent stability.

\subsection{PET process between $\beta-C D-M o S_{2} Q D s$ and $p-N P$}

According to the previous study [36], p-NP is capable to enter the $\beta$-CD cavity to form a host-guest inclusion because of the large binding constant $\left(2.3 \times 10^{3} \mathrm{M}^{-1}\right)$ [37]. This process can close the distance between $\mathrm{p}-\mathrm{NP}$ and $\beta$-CD-MoS 2 QDs, and can quench the fluorescence of $\beta$-CD-MoS $\mathrm{S}_{2}$ QDs. As shown in Fig. 3A, the fluorescence intensity of $\beta$-CD-MoS $\mathrm{S}_{2}$ QDs dramatically decreased with the addition of p-NP, which was consistent with resonance energy transfer (RET) or photo-induced electron transfer (PET) process [38]. For distinguishing whether the quenching effect is resulted from RET or PET, the fluorescence lifetime decay curve was further measured. As illustrated in Fig. 3B, the fluorescence lifetime of $\beta$-CD-MoS 2 QDs shows significant shorter after the addition of $\mathrm{p}-\mathrm{NP}$, which certificated that the quenching mechanism is related to PET [39]. Moreover, the absorption spectrum from $\mathrm{p}-\mathrm{NP}$ and emission spectrum from $\beta$-CD-MoS $\mathrm{MDs}_{2}$ Q verifies the hypothesis, as from Fig. S6, almost no overlap presents between the emission spectrum from $\beta$-CD-MoS 2 QDs and absorption spectrum from $\mathrm{p}-\mathrm{NP}$, which is inconsistent with the resonance energy transfer (RET) mechanism [40]. Therefore, the fluorescence quenching mechanism of $\mathrm{p}$-NP for $\beta$-CD-MoS $\mathrm{S}_{2}$ QDs is attributed to PET process. Furthermore, an obviously blue-shift in fluorescence of $\beta$-CD-MoS 2 QDs is observed in the presence of $\mathrm{p}-\mathrm{NP}$. The blue shift might be attribute to the influence via host-guest interaction between $\mathrm{p}-\mathrm{NP}$ and $\beta$-CD on the optical property of nanoprobe [41].

\subsection{Fluorescence detection of MP based on $\beta$-CD-MoS 2 QDs}

Based on the feature that MP can be hydrolyzed to produce p-NP under alkaline condition, and then the resulting p-NP can quench efficiently the fluorescence of $\beta$-CD-MoS $\mathrm{S}_{2} \mathrm{QDs}$, the fluorescence detection of MP was carried out based on the $\beta$-CD-MoS ${ }_{2}$ QDs nanoprobe. Firstly, the feasibility of the proposal was studied and the related results are exhibited in Fig. 4. For pure $\mathrm{MoS}_{2}$ QDs (Fig. 4A), its fluorescence intensity only shows a very slight decrease in the presence of hydrolyzed MP. As for $\beta$-CD-MoS 2 QDs (Fig. 4B), its fluorescence intensity barely changed in the presence or absence of MP; however, it can be dramatically quenched in the present of hydrolyzed MP, suggesting the produced p-NP from the hydrolysis reaction of MP can entry into the $\beta$-CD cavity to quench the fluorescence intensity of $\beta$-CD-MoS 2 QDs. These results demonstrate that the fluorescence detection of MP based on $\beta$-CD-MoS2 QDs is feasible.

To realize the best performance of $\beta$-CD-MoS 2 QDs for detecting MP, some experiment conditions were optimized. As illustrated in Fig. S7A, the absorbance intensity of MP at $400 \mathrm{~nm}$ reached the highest after the addition of $35 \mathrm{mM} \mathrm{NaOH}$. However, as exhibited in Fig. S8, the fluorescence intensity of $\beta$-CD-MoS 2 QDs is dramatically decreased when the solution has a strongly alkaline $(\mathrm{pH}=10.0)$. This phenomenon might be due to the broken of amide bond, and the $\beta$-CD molecules cannot connect with $\mathrm{MoS}_{2}$ QDs. Thus, the $\mathrm{pH}$ of hydrolyzed MP solution was adjusted to neutral. Furthermore, the optimum for incubation time and temperature were also studied. As shown in Fig. S7B, the $\left(\mathrm{F}_{0}-\mathrm{F}\right) / \mathrm{F}_{0}\left(\mathrm{~F}_{0}\right.$ represents the background fluorescence of $\beta-\mathrm{CD}-\mathrm{MoS}_{2}$ without MP, and F represents the fluorescence of $\beta$-CD-MoS2 with different MP concentrations) reaches a maximum when the reaction time reached $20 \mathrm{~min}$. It is noted that the quenching efficiency reaches a maximum when the temperature of the system is $60^{\circ} \mathrm{C}$ (Fig. S7C). Based on these observations, the analyte system was incubated at $70{ }^{\circ} \mathrm{C}$ for $20 \mathrm{~min}$ before the measurement for further experiments.

Under the optimum conditions, the usability of this fluorescent nanoprobe for sensitive detection of MP was measured. Fig. 5A shows the fluorescence intensity of $\beta$-CD-MoS ${ }_{2}$ QDs gradually decreases with MP concentrations increasing from $0.01 \mathrm{ppm}$ to $36.0 \mathrm{ppm}$. It can be observed a good linear relationship is presented between the fluorescence intensity and concentrations of MP ranging from $0.01 \mathrm{ppm}$ to 18.0 ppm (Fig. 5B). The regression equation can be expressed as $\left(\mathrm{F}_{0}-\mathrm{F}\right) / \mathrm{F}_{0}=$ $0.059[\mathrm{MP}]+0.085$, where $\mathrm{R}^{2}$ is 0.992 . The detection limit was estimated to be $3.3 \mathrm{ppb}(\mathrm{S} / \mathrm{N}=3)$. A comparison to previous methods for MP detection is shown in Table S1, which suggests that the $\beta-\mathrm{CD}-\mathrm{MoS}_{2}$ QDs based assay exhibits lower detection limit and wider linear range. More importantly, the proposed method is the first example to achieve sensitive detection of MP using a $\beta$-CD modified $\mathrm{MoS}_{2}$ QDs through hostguest recognition without the addition of enzyme.
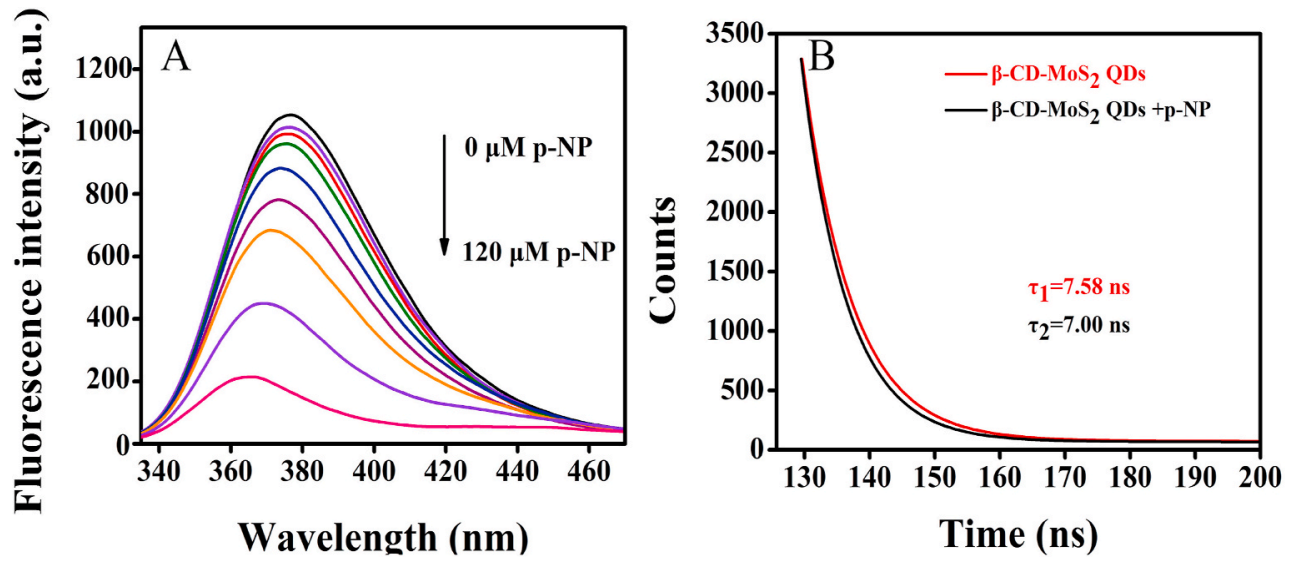

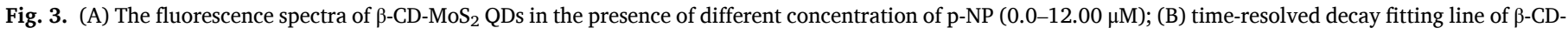
$\mathrm{MoS}_{2}$ QDs in the absence and presence of p-NP $(100 \mu \mathrm{M})$. 

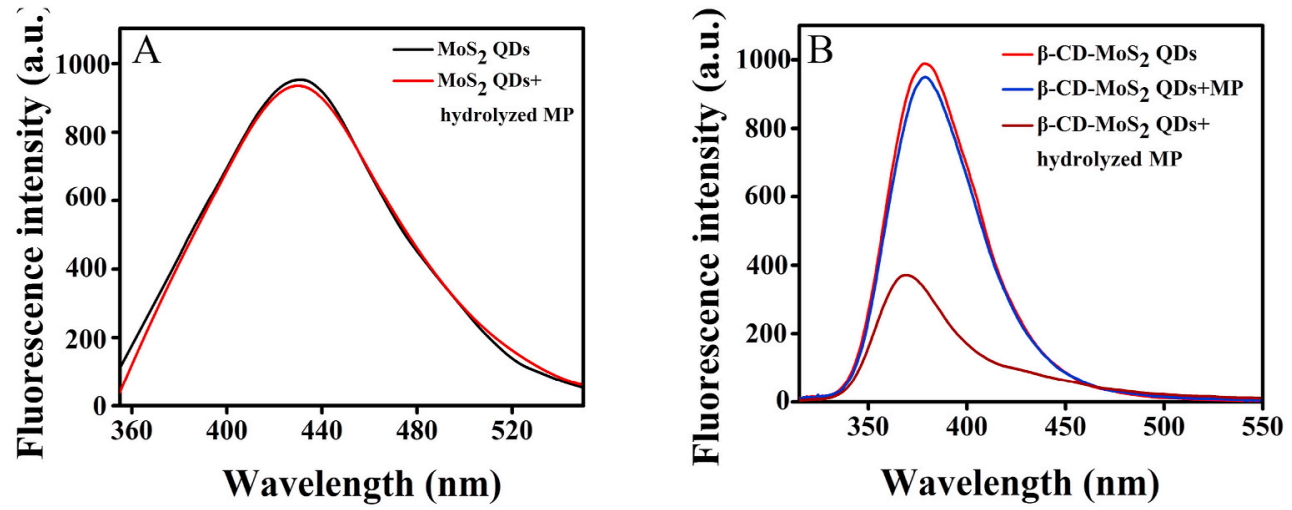

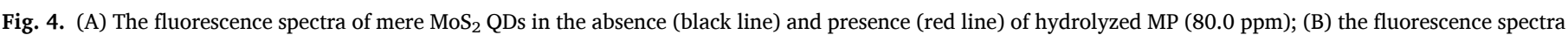

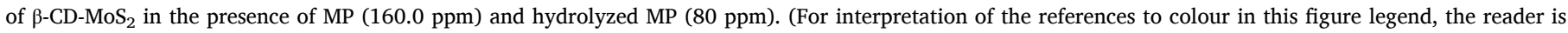
referred to the Web version of this article.)
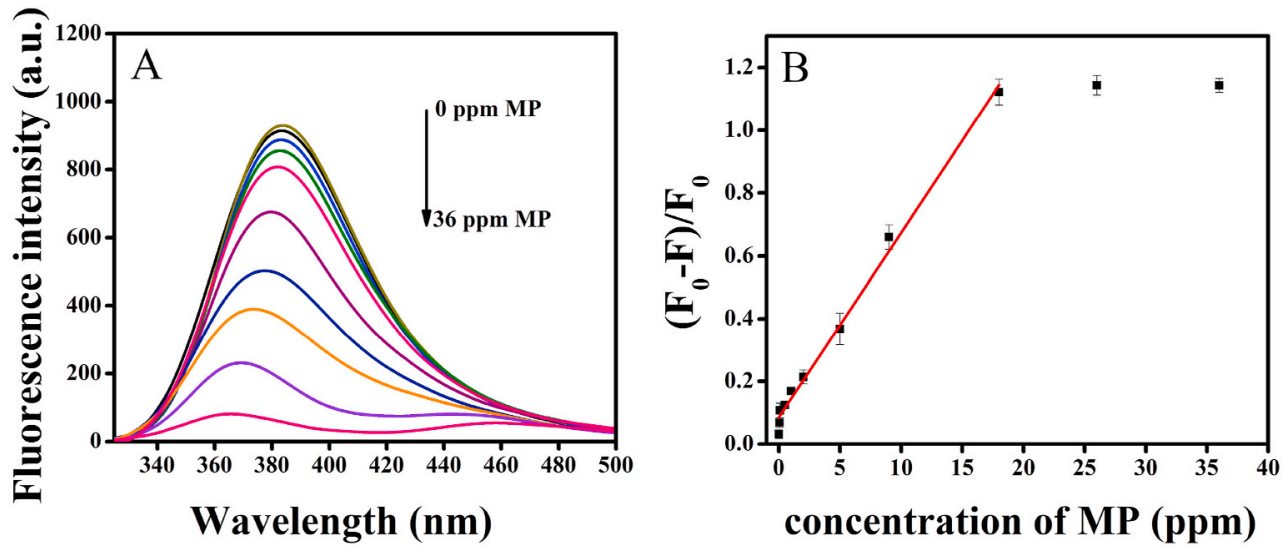

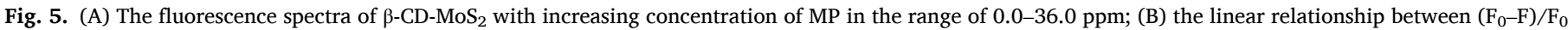

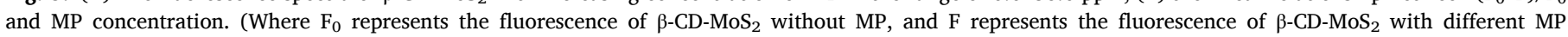
concentration).

\subsection{Selectivity and real application}

To validate the selectivity of the proposed proposal for the determination of MP, the other organophosphorus pesticides (triazophos, dibrom, phosphamidon, glyphosate, methamidophos, dimethoate, phoxim, systox, and trichlorfon) were also analyzed using the $\beta$-CD$\mathrm{MoS}_{2}$ QDs fluorescent nanoprobe. As shown in Fig. S9, the fluorescence quenching efficiency of $\beta$-CD-MoS 2 QDs by MP $(20.0 \mathrm{ppm})$ is prominent higher than other pesticides with relatively higher concentration (100.0 $\mathrm{ppm}$ ). These results demonstrate that the proposed method is highly selective for distinguishing MP from other organophosphorus pesticides. Next, the practicality of the fluorescence nanoprobe for MP detection was investigated in real sample analysis including fruit and vegetable. Different concentration of MP (0.0, 1.0, 5.0 and $10.0 \mathrm{ppm})$ were spiked with processed apple and cucumber samples, the fluorescence was measured in a similar experiment procedure, and the results exhibit good recoveries ranging from $93.0 \%$ to $105.6 \%$ with a maximum RSD of $4.42 \%$ (Table S2), indicating that the proposed fluorescent nanoprobe has important potential applicability for the determination of MP in real samples.

\section{Conclusions}

In summary, this work developed an enzyme-free fluorescent nanoprobe based on $\beta$-CD-MoS 2 QDs for MP detection through hostguest recognize for the first time. By introducing $\beta$-CD molecules on the $\mathrm{MoS}_{2}$ QDs surface, the formed novel $\beta$-CD-MoS 2 QDs nanoprobe can exhibit the synthetic advantages from each component (e.g., excellent optical property of $\mathrm{MoS}_{2}$ QDs and high molecular recognition performance of $\beta$-CD). Via the PET process, the p-NP molecules from MP hydrolysis under alkaline conditions can entry into the inner cavities of $\beta$-CD owing to its host-guest recognition and further quench the fluorescence of $\beta-\mathrm{CD}-\mathrm{MoS}_{2}$ QDs, thus a fluorescence sensing platform was constructed for MP. After optimizing various experimental conditions, the results show that the $\beta-\mathrm{CD}-\mathrm{MoS}_{2}$ QDs fluorescent nanoprobe presents excellent selectivity and sensitivity with wide linear range (three orders of magnitude) and low detection limit (3.3 ppb) toward MP detection. Furthermore, the developed nanoprobe can be applied successfully to the real food samples for MP analysis. It's believed that the $\beta-\mathrm{CD}-\mathrm{MoS}_{2}$ QDs probe can give a new sight for the selective and sensitive detection of MP and has potential for chem/biological analysis.

\section{Credit author statement}

Yinhui Yi: Supervision, Conceptualization, Methodology, Experiment, Writing- Reviewing and Editing. Wei Zeng: Experiment, Data curation, Writing-Editing. Gangbing Zhu: Validation, Writing- Reviewing and Editing.

\section{Declaration of competing interest}

The authors declare that they have no known competing financial interests or personal relationships that could have appeared to influence the work reported in this paper. 


\section{Acknowledgments}

This work is supported by the National Natural Science Foundation of China (21607061), the CAS Key Laboratory of Coastal Environmental Processes and Ecological Remediation, YICCAS (Grant No.: 2020KFJJ16), the Opening Project of State Key Laboratory of Chemo/ Biosensing and Chemometrics of Hunan University (2018019), the Open Project Program of Key Laboratory for Analytical Science of Food Safety and Biology, Ministry of Education (No. FS2008) and the Priority Academic Program Development of Jiangsu Higher Education Institutions, Collaborative Innovation Center of Technology and Material of Water Treatment.

\section{Appendix A. Supplementary data}

Supplementary data to this article can be found online at https://doi. org/10.1016/j.talanta.2020.121703.

\section{References}

[1] A. Samsidar, S. Siddiquee, S.M. Shaarani, A review of extraction, analytical and advanced methods for determination of pesticides in environment and foodstuffs, Trends Food Sci. Technol. 71 (2018) 188-201.

[2] J.-W. Zhou, X.-M. Zou, S.-H. Song, G.-H. Chen, Quantum dots applied to methodology on detection of pesticide and veterinary drug residues, J. Agric. Food Chem. 66 (2018) 1307-1319.

[3] B. Liu, G. Han, Z. Zhang, R. Liu, C. Jiang, S. Wang, M.-Y. Han, Shell thicknessdependent Raman enhancement for rapid identification and detection of pesticide residues at fruit peels, Anal. Chem. 84 (2012) 255-261.

[4] W. Zhu, W. Liu, T. Li, X. Yue, T. Liu, W. Zhang, S. Yu, D. Zhang, J. Wang, Facile green synthesis of graphene-Au nanorod nanoassembly for on-line extraction and sensitive stripping analysis of methyl parathion, Electrochim. Acta 146 (2014) 419-428.

[5] C. Esen, J. Czulak, T. Cowen, E. Piletska, S.A. Piletsky, Highly efficient abiotic assay formats for methyl parathion: molecularly imprinted polymer nanoparticle assay as an alternative to enzyme-linked immunosorbent assay, Anal. Chem. 91 (2019) 958-964.

[6] S. Lan, X. Wang, Q. Liu, J. Bao, M. Yang, H. Fa, C. Hou, D. Huo, Fluorescent sensor for indirect measurement of methyl parathion based on alkaline-induced hydrolysis using N-doped carbon dots, Talanta 192 (2019) 368-373.

[7] H. Wu, Y. Luo, C. Hou, D. Huo, Y. Zhou, S. Zou, J. Zhao, Y. Lei, Flexible bipyramidAuNPs based SERS tape sensing strategy for detecting methyl parathion on vegetable and fruit surface, Sensor. Actuator. B Chem. 285 (2019) 123-128.

[8] Y. Li, J. Liu, Y. Zhang, M. Gu, D. Wang, Y.Y. Dang, B.C. Ye, Y. Li, A robust electrochemical sensing platform using carbon paste electrode modified with molecularly imprinted microsphere and its application on methyl parathion detection, Biosens. Bioelectron. 106 (2018) 71-77.

[9] C. Zhang, H. Cui, Y. Han, F. Yu, X. Shi, Development of a biomimetic enzymelinked immunosorbent assay based on molecularly imprinted polymers on paper for the detection of carbaryl, Food Chem. 240 (2018) 893-897.

[10] H. Li, X. Yan, G. Lu, X. Su, Carbon dot-based bioplatform for dual colorimetric and fluorometric sensing of organophosphate pesticides, Sensor. Actuator. B Chem. 260 (2018) 563-570.

[11] F. Zhang, Y. Liu, P. Ma, S. Tao, Y. Sun, X. Wang, D. Song, A Mn-doped ZnS quantum dots-based ratiometric fluorescence probe for lead ion detection and "off-on" strategy for methyl parathion detection, Talanta 204 (2019) 13-19.

[12] X. Yan, H. Li, Y. Yan, X. Su, Selective detection of parathion-methyl based on nearinfrared CuInS 2 quantum dots, Food Chem. 173 (2015) 179-184.

[13] S. Huang, J. Yao, X. Chu, Y. Liu, Q. Xiao, Y. Zhang, One-Step Facile Synthesis of nitrogen-doped carbon dots: a ratiometric fluorescent probe for evaluation of acetylcholinesterase activity and detection of organophosphorus pesticides in tap water and food, J. Agric. Food Chem. 67 (2019) 11244-11255.

[14] A. Zheng, C. Shen, Q. Tang, C.-B. Gong, C.-F. Chow, Catalytic chemosensing assay for selective detection of methyl parathion organophosphate pesticide, Chem. Eur J. 25 (2019) 9643-9649.

[15] L. Liu, G. Zhu, W. Zeng, B. Lv, Y. Yi, Highly sensitive and selective "off-on" fluorescent sensing platform for $\mathrm{ClO}-$ in water based on silicon quantum dots coupled with nanosilver, Anal. Bioanal. Chem. 411 (2019) 1561-1568.

[16] K. Zhang, H. Zhou, Q. Mei, S. Wang, G. Guan, R. Liu, J. Zhang, Z. Zhang, Instant visual detection of trinitrotoluene particulates on various surfaces by ratiometric fluorescence of dual-emission quantum dots hybrid, J. Am. Chem. Soc. 133 (2011) 8424-8427.
[17] H. Zhu, H. Zhang, Y. Xia, Planar Is Better: Monodisperse three-Layered $\mathrm{MoS}_{2}$ quantum dots as fluorescent reporters for 2,4,6-trinitrotoluene sensing in environmental water and luggage cases, Anal. Chem. 90 (2018) 3942-3949.

[18] W. Dai, H. Dong, B. Fugetsu, Y. Cao, H. Lu, X. Ma, X. Zhang, Tunable fabrication of molybdenum disulfide quantum dots for intracellular microRNA detection and multiphoton bioimaging, Small 11 (2015) 4158-4164.

[19] F. Sarpong, P. Otengdarko, M.K. Golly, L.P. Amenorfe, M.T. Rashid, C. Zhou, Comparative study of enzymes inactivation and browning pigmentation of apple (Malus domestica) slices by selected gums during low temperature storage, J. Food Biochem. 42 (2018).

[20] W. Gu, Y. Yan, C. Zhang, C. Ding, Y. Xian, One-step synthesis of water-soluble MoS quantum dots via a hydrothermal method as a fluorescent probe for hyaluronidase detection, ACS Appl. Mater. Interfaces 8 (2016) 11272-11279.

[21] J. Ma, H. Yu, X. Jiang, Z. Luo, Y. Zheng, High sensitivity label-free detection of Fe3 + ion in aqueous solution using fluorescent MoS2 quantum dots, Sensor. Actuator. B Chem. 281 (2019) 989-997.

[22] R. Zeng, Z. Luo, L. Zhang, D. Tang, Platinum nanozyme-catalyzed gas generation for pressure-based bioassay using polyaniline nanowires-functionalized graphene oxide framework, Anal. Chem. 90 (2018) 12299-12306.

[23] Z. Wang, J. Lin, J. Gao, Q. Wang, Two optically active molybdenum disulfide quantum dots as tetracycline sensors, Mater. Chem. Phys. 178 (2016) 82-87.

[24] G.M. Durán, C. Abellán, A.M. Contento, Á. Ríos, Discrimination of penicillamine enantiomers using $\beta$-cyclodextrin modified CdSe/ZnS quantum dots, Microchimica Acta 184 (2017) 815-824.

[25] Y. Yi, D. Zhang, Y. Ma, X. Wu, G. Zhu, Dual-signal electrochemical enantiospecific recognition system via competitive supramolecular host-guest interactions: the case of phenylalanine, Anal. Chem. 91 (2019) 2908-2915.

[26] A. Mondal, N.R. Jana, Fluorescent detection of cholesterol using beta-cyclodextrin functionalized graphene, Chem. Commun. 48 (2012) 7316-7318.

[27] J. Zhang, J. Wang, L. Yang, B. Liu, G. Guan, C. Jiang, Z. Zhang, Ligand replacement induced chemiluminescence for selective detection of an organophosphorus pesticide using bifunctional $\mathrm{Au}-\mathrm{Fe}_{3} \mathrm{O}_{4}$ dumbbell-like nanoparticles, Chem. Commun. 50 (2014) 15870-15873.

[28] Ronit Freeman, Tali Finder, LiLy Bahshi, I. Willner, $\beta$-cyclodextrin-modified CdSe/ ZnS quantum dots for sensing and chiroselective analysis, Nano Lett. 9 (2009) 2073-2076.

[29] Mai Luo, Yifan Hua, Yiran Liang, Jiajun Han, Donghui Liu, Wenting Zhao, P. Wang, Synthesis of novel $\beta$-cyclodextrin functionalized S, N codoped carbon dots for selective detection of testosterone, Biosens. Bioelectron. 98 (2017) 195-201.

[30] C. Tang, Z. Qian, Y. Huang, J. Xu, H. Ao, M. Zhao, J. Zhou, J. Chen, H. Feng, A fluorometric assay for alkaline phosphatase activity based on beta-cyclodextrinmodified carbon quantum dots through host-guest recognition, Biosens. Bioelectron. 83 (2016) 274-280.

[31] Wei Zeng, Lirong Liu, Yinhui Yi, Yuntao Wu, Ning Sun, Baohe Lv, G. Zhu, A double signal nanoprobe based on molybdenum disulfide quantum dots, Microchem. J. 150 (2019).

[32] S. Xu, D. Li, P. Wu, One-pot, facile, and versatile synthesis of monolayer $\mathrm{MoS}_{2} / \mathrm{WS}_{2}$ quantum dots as bioimaging probes and efficient electrocatalysts for hydrogen evolution reaction, Adv. Funct. Mater. 25 (2015) 1127-1136.

[33] M. Li, L. Zhang, X. Fan, M. Wu, Y. Du, M. Wang, Q. Kong, L. Zhang, J. Shi, Dual synergetic effects in MoS 2/pyridine-modified g- $\mathrm{C}_{3} \mathrm{~N}_{4}$ composite for highly active and stable photocatalytic hydrogen evolution under visible light, Appl. Catal. B Environ. 190 (2016) 36-43.

[34] X. Zhou, X. Gao, F. Song, C. Wang, F. Chu, S. Wu, A sensing approach for dopamine determination by boronic acid-functionalized molecularly imprinted graphene quantum dots composite, Appl. Surf. Sci. 423 (2017) 810-816.

[35] S. Xu, S. Che, P. Ma, F. Zhang, L. Xu, X. Liu, X. Wang, D. Song, Y. Sun, One-step fabrication of boronic-acid-functionalized carbon dots for the detection of sialic acid, Talanta 197 (2019) 548-552.

[36] Zhixing Zhang, Jie Zhou, Yun Liu, J. Tang, W. Tang, Cyclodextrin capped CdTe quantum dots as versatile fluorescence sensors for nitrophenol isomers, Nanoscale 7 (2015) 19540-19546.

[37] Q. Sun, S. Fang, Y. Fang, Z. Qian, H. Feng, Fluorometric detection of cholesterol based on beta-cyclodextrin functionalized carbon quantum dots via competitive host-guest recognition, Talanta 167 (2017) 513-519.

[38] L. Jia, J.P. Xu, D. Li, S.P. Pang, Y. Fang, Z.G. Song, J. Ji, Fluorescence detection of alkaline phosphatase activity with beta-cyclodextrin-modified quantum dots, Chem. Commun. 46 (2010) 7166-7168.

[39] Z.-Y. Lin, Y.-C. Kuo, C.-J. Chang, Y.-S. Lin, T.-C. Chiu, C.-C. Hu, Highly sensitive sensing of hydroquinone and catechol based on $\beta$-cyclodextrin-modified carbon dots, RSC Adv. 8 (2018) 19381-19388.

[40] M.I. Halawa, F. Wu, T.H. Fereja, B. Lou, G. Xu, One-pot green synthesis of supramolecular -cyclodextrin unctionalized gold nanoclusters and their application for highly selective and sensitive fluorescent detection of dopamine, Sensor. Actuator. B Chem. 254 (2018) 1017-1024.

[41] C. Tang, J. Zhou, Z. Qian, Y. Ma, Y. Huang, H. Feng, A universal fluorometric assay strategy for glycosidases based on functional carbon quantum dots: $\beta$-galactosidase activity detection in vitro and in living cells, J. Mater. Chem. B 5 (2017) 1971-1979. 